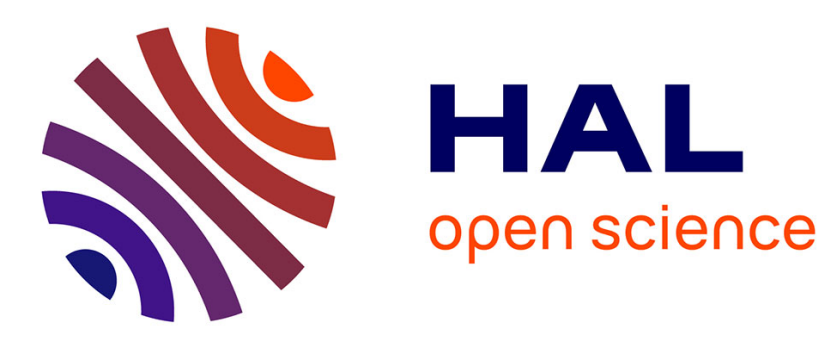

\title{
Composition, Chemical Variability and Biological Activity of Cymbopogon schoenanthus Essential Oil from Central Algeria
}

Charaf Eddine Watheq Malti, Imad Abdelhamid El Haci, Faiçal Hassani, Mathieu Paoli, Marc Gibernau, Félix Tomi, Joseph Casanova, Chahrazed Bekhechi

\section{To cite this version:}

Charaf Eddine Watheq Malti, Imad Abdelhamid El Haci, Faiçal Hassani, Mathieu Paoli, Marc Gibernau, et al.. Composition, Chemical Variability and Biological Activity of Cymbopogon schoenanthus Essential Oil from Central Algeria. Chemistry and Biodiversity, 2020, 17 (6), 10.1002/cbdv.202000138 . hal-02999044

\section{HAL Id: hal-02999044 \\ https://hal.science/hal-02999044}

Submitted on 12 Nov 2020

HAL is a multi-disciplinary open access archive for the deposit and dissemination of scientific research documents, whether they are published or not. The documents may come from teaching and research institutions in France or abroad, or from public or private research centers.
L'archive ouverte pluridisciplinaire HAL, est destinée au dépôt et à la diffusion de documents scientifiques de niveau recherche, publiés ou non, émanant des établissements d'enseignement et de recherche français ou étrangers, des laboratoires publics ou privés. 


\title{
Composition, chemical variability and biological activity of Cymbopogon schoenanthus essential oil from Central Algeria
}

\author{
Charaf Eddine Watheq Maltia , Imad Abdelhamid El Haci ${ }^{\mathrm{a}, \mathrm{b}}$, Faiçal Hassanic, Mathieu Paolid, \\ Marc Gibernau ${ }^{\mathrm{d}}$, Félix Tomi ${ }^{*, \mathrm{~d}}$, Joseph Casanova ${ }^{\mathrm{d}}$, Chahrazed Bekhechi ${ }^{\mathrm{a}}$
}

\begin{abstract}
aLaboratoire des Produits Naturels, Département de Biologie, Université Abou Bekr Belkaïd, 13000 Tlemcen, Algeria.
${ }^{b}$ Centre de Recherche scientifique et technique en Analyses Physico-chimiques (CRAPC), Bou-lsmail, 42000 Tipaza, Algérie
\end{abstract}

'Laboratoire d'Ecologie et Gestion des Ecosystèmes Naturels, Département d'Ecologie et Environnement, Université Abou Bekr Belkaïd, 13000 Tlemcen, Algeria,

dUniversité de Corse-CNRS, UMR 6134 SPE, Equipe Chimie et Biomasse, Route des Sanguinaires, 20000 Ajaccio, France (tomi_f@univ-corse.fr)

\begin{abstract}
Cymbopogon schoenanthus (L.) Sprengel (Poaceae) is an aromatic plant whose aerial parts and rhizome produced an essential oil with pleasant odor. A chemical variability has been observed depending of the countries where the plant grows wild, including Algeria. The chemical compositions of 24 oil samples isolated from plants harvested in Central Algeria have been investigated. Twenty of these were dominated by cis-and trans-p-menth-2en-1-ols $(22.6 \% \pm 3.6$ and $14.3 \% \pm 1.7$, respectively) beside four atypical compositions. Otherwise, aerial parts and rhizomes produced similar essential oils. Lastly, a fair antimicrobial activity was measured against Staphylococcus aureus strain, while the antioxidant potential was low.
\end{abstract}

Keywords: Cymbopogon schoenanthus • essential oil composition • chemical variability • antimicrobial activity • Algeria

\section{Introduction}

The genus Cymbopogon is comprised of 144 species and it is widely distributed in the tropical and subtropical regions of Africa, Asia and America. ${ }^{[1]}$ Beside C. flexuosus and C. citratus (lemongrass), commercially cultivated for their essential oils, Cymbopogon schoenanthus (L.) Sprengel (Poaceae) grows wild in most tropical countries of Africa and Asia. ${ }^{[2-4]}$. It is known since the antiquity. Indeed, when the tombs of certain Egyptian kings of the 20th and 21st dynasties (3,000 years ago) were opened in 1881, the scent of Cymbopogon schoenanthus grass was readily detected by smell. ${ }^{[5]}$

The species Cymbopogon schoenanthus (L.) Sprengel is constituted of two sub-species, C. schoenanthus ssp. schoenanthus [synonyms = ssp. laniger (Desf.) Maire \& Weiller; Andropogon schoenanthus Linnaeus] and ssp. proximus (Hochst. ex A. Rich.) Maire \& Weiller. We will focus our study on C. schoenanthus ssp. schoenanthus.

Cymbopogon schoenanthus is a compact, evergreen, perennial grass forming dense tussocks at the base and numerous erect $60-80 \mathrm{~cm}$ stems. The leaves are linear and strongly curved. Each spike contains a single flower. Flowering takes place in the spring. The roots have a pleasant aromatic smell. The plant grows in arid regions and it is found on the gravelly-stony soils of djebels.

Cymbopogon schoenanthus is used as traditional medicine to treat digestive diseases: aerophagia, flatulence and urinary decrease, analeptic drink for new mother after childbirth, bad breath, gumboils and urinary incontinence. ${ }^{[6]}$ In the Djanet area, the young leaves are eaten with a salad or cooked with meat. Straw was used in the Sahara to fill mattresses.

Various organs of the plant produce an essential oil: aerial parts, including leaves, stems, and flowers (inflorescences) as well as roots. Their composition has been investigated and demonstrated a strong variability. For instance:

- Commercial C. schoenanthus oil sample analyzed in Brazil contained mainly geraniol (62.5\%) followed by geranial and neral; ${ }_{i}^{[7]}$

- The major constituents of C. schoenanthus leaf oil from Indian Thar Desert were found to be 2-undecanone (14.68\%) and limonene (19.54\%); 
- Wild and cultivated aerial parts of $C$. schoenanthus from Uttarakhand region North Himalaya produced an essential oil containing cis-sabinene hydrate (30.1 and 31.6\%), trans-sabinene hydrate (17.1 and 14.5\%), cis-piperitol (9.6 and 18.5\%), trans-piperitol (8.5 and 7.2\%) beside piperitone, limonene and pcymene; $^{[9]}$

- An oil sample from Saudi Arabia contained, as major components, piperitone (14.6\%), $\beta$-elemene (11.6\%), $\alpha$-eudesmol (11.5\%), elemol (10.8\%), $\beta$ eudesmol (8.5\%) beside the unusual cyclohexanemethanol (11.6\%) and 2-naphthalenemethanol (7.1\%); ${ }^{[10]}$

- In contrast, the composition of an oil sample of Iranian origin (aerial parts) was dominated by piperitone (62.0\%) beside $\alpha$-terpinene $(7.1 \%) .{ }^{[11]}$

Piperitone was the major component of $C$. schoenanthus oils from central Africa. For instance, it accounted for 58.9-68.5\% in Beninese oils, followed by $\delta$-2-carene (11.5-15.5\%). ${ }^{[12-16]}$ High contents of piperitone were also found in Togolese oils (61.0-69.8\%) followed by $\delta$-2-carene (15.1-23.4\%; $5.4 \%$ one sample) and sometimes by $\beta$-elemol and eremoligenol. ${ }^{[17-22]} \mathrm{An}$ oil sample from Burkina Faso contained piperitone (42.0\%), 2-carene (8.2\%), elemol (6.2\%). ${ }^{[23]}$ Another Burkinabe oil sample contained piperitone at lesser extent (18.48\%) beside elemol (18.3\%), eudesm-7(11)-en-4-ol (17.1\%), $\alpha$ eudesmol (10.7\%).[24] Piperitone-rich oils have been also reported in Sudan (47.7-71.5\%) beside $\delta$-2-carene (4.5-10.0\%), $\beta$-elemol (2.7-9.0\%) and/or intermedeol (6.1-17.3\%). ${ }^{[25]}$

In contrast with the compositions of African C. schoenanthus oils reported below, two oil samples isolated from plants harvested in Southern Tunisia displayed quite different compositions (leaves, 6 samples/roots, 2 samples, \%): limonene, 22.1-27.3/26.0-10.5; $\beta$-phellandrene (13.4-16.3/15.98.2), $\delta$-terpinene (7.4- 21.2/9.7-4.3), $\alpha$-terpineol (9.1-11.7/9.4-6.8), $\beta$-eudesmol (0.2-5.3/2.1-14.2), $\alpha$-eudesmol (0.4-5.5/4.3-0.3), juniper camphor (2.74.1/3.3-8.2) ${ }^{[26]}$

Among African countries, Algeria is an immense territory stretched from the Mediterranean coast to the arid lands of Southern Sahara. Wild $C$. schoenanthus has been reported in various regions from North to South Algeria and recently the compositions of several oil samples have been reported, evidencing a chemical variability:

- The first study dates back to 2005 and it concerned a supercritical $\mathrm{CO}_{2}$ extract. The main components were cis-p-menth-2-en-1-ol (17.78\%), trans-pmenth-2-en-1-ol (10.83\%) (Both not yet mentioned in other countries), beside $\beta$-elemol (13.71\%), cis-piperitol (9.14\%) and trans-piperitol (6.26\%):[27]

- An essential oil sample from Ain Sefra region (about $250 \mathrm{Km}$ South of Tlemcen city, dried leaves), confirmed cis-p-menth-2-en-1-o1 (18.6), trans- $p$ menth-2-en-1-ol (9.5\%), as major components beside elemol (7.4\%), piperitone (7.2\%) and limonene (7.0\%); ${ }^{[2]}$

- A similar composition was reported for an essential oil sample isolated from aerial parts harvested in Tébessa region, Eastern Algeria (cis-p-menth-2en-1-ol 15\%, trans-p-menth-2-en-1-ol 13\%, trans-piperitol 9\%, cis-piperitol 8\%, 2-carene 7\%); ${ }^{[29]}$

- Menthenols were the main components of essential oils isolated from plants harvested in El Menia, Béchar, Ghardaïa and Ouargla regions (cis-pmenth-2-en-1-ol 12.1-15.1\%, trans-p-menth-2-en-1-ol 6.7-9.2\%, trans-piperitol 5.4-7.7\%, cis-piperitol 3.3-6.9\%, 2-carene 13.4-20.4\%, limonene 0-13.0\%, elemol 4.9-9.9\%, $\beta$-eudesmol 2.8-7.7\%); ${ }^{[30]}$

- In contrast, essential oil isolated from plants harvested in Southern Algeria contained piperitone as main component. Indeed, piperitone reached $63.3 \%$ in oil from Illizi province, near Djanet, followed by $\beta$-eudesmol $9.3 \%$ and elemol $6.9 \%{ }^{[31]}$ Similarly, the contents of piperitone were 63.2 and $55.1 \%$ in oils from Djanet and Tamanrasset, respectively, accompanied by 2-carene (9.3 and 16.7\%) and elemol (9.5 and 4.8\%); ${ }^{[30]}$

- Lastly, totally different compositions have been recently reported for oil samples isolated from aerial parts and roots of plant harvested in Ghardaïa region: guaiol (20.4\%), cis- $\beta$-terpineol (16.2\%), hinesol (10.5\%), cis-sabinene hydrate (10.0\%) for aerial part oil; and agarospirol (14.2\%), cis- $\beta$-terpineol (12.6\%), 4-carene (6.9\%), cis-sabinene hydrate (6.6\%) and guaiol (5.9\%), for root oil. ${ }^{[32]}$ Leaves collected from Oued Sbaa, El-Menia (Ghardaïa and at 267 km South-West of Ghardaïa, respectively) contained a-eudesmol (17.9\%), trans-dauca-4(11),7-diene (10.7\%), a-muurolol (= $\delta$-cadinol, $6.6 \%)$, hinesol $(6.1 \%)^{[33]}$

Obviously, this review of the literature evidenced a chemical variability within C. schoenanthus essential oils, including Algerian oils. In short, the compositions of $C$. schoenanthus oils from Southern Algeria (Illizi and Tamanrasset provinces) were dominated by piperitone, like oils from Benin, Togo, Burkina Faso or Sudan. In contrast, oils from central to Northern Algeria (Béchar, Ghardaïa, Ouargla, Aïn Sefra and Tébessa provinces) contained mainly cis- and trans-p-menth-2-en-1-ols, not identified in C. schoenanthus oils from other countries (India, Saudi Arabia, Iran, Tunisia). It could be highlighted that menth-2-en-1-ol isomers bring some originality to Algerian C. schoenanthus oils.

However, the composition of only one or two oil samples was reported for every location of harvest and therefore, it was not possible to evidence homogeneity or a chemical variability within a given locality. The knowledge of homogeneity/chemical variability is crucial for an eventual commercialization of Algerian C. schoenanthus essential oil. Therefore, the aim of the present study was to isolate and to analyze a sufficiently large number of oil samples to evidence homogeneity or chemical variability within a location of harvest.

\section{Results and Discussion}


Two locations of harvest have been chosen in central Algeria, in provinces of Béchar and Ghardaïa. The whole plant (aerial parts and rhizomes) has been used to isolate essential oil. Indeed, we observed that it is easier to harvest the whole plant than pick up aerial parts without rhizomes. Therefore, our work has been divided as follow:

1. Analysis of three oil samples by combination of chromatographic and spectroscopic techniques (GC/RI, GC/MS, $\left.{ }^{13} \mathrm{C}-\mathrm{NMR}\right)$ in order to identify as much as possible components;

2. Comparison of oil samples isolated separately from aerial parts and rhizomes of the same individual plant ( 6 samples);

3. Analysis of 24 oil samples isolated from plants (whole plant) harvested in Béchar (10 samples) and in Ghardaïa (14 samples);

4. Antimicrobial activity, antioxidant activity.

Detailed analysis of three oil samples

Three C. schoenanthus oil samples isolated from the whole plant, one sample from Béchar, the two other from Ghardaïa, have been submitted to GC in combination with Retention Indices on two columns of different polarity, GC/MS and ${ }^{13} \mathrm{C}-\mathrm{NMR}$, following a computerized method developed at the University of Corsica. ${ }^{[34-36]}$ In total, 48 compounds have been identified. The compositions of the three samples were dominated by various alcohols bearing the $p$-menthane skeleton: cis-p-menth-2-en-1-ol (22.6-28.5\%), trans-p-menth-2-en-1-ol (15.4-16.3\%), cis- and trans-piperitols (5.4-8.5\% and 8.8$8.9 \%$, respectively). Monoterpene hydrocarbons were represented by $\delta$-2-carene (8.6-17.0\%) and limonene (2.4-5.9\%). $\beta$-Elemol (5.6-7.4\%) was the main oxygenated sesquiterpene accompanied by $\beta$-eudesmol, $\alpha$-eudesmol, intermedeol and juniper camphor (up to $3.5 \%$ each).

Table 1: Chemical composition of three oil samples isolated from the whole plant of $C$. schoenanthus.

\begin{tabular}{|c|c|c|c|c|c|c|}
\hline Compounds ${ }^{[a]}$ & $\mathbf{R l}^{\left[{ }^{[b]}\right]}$ & $\mathbf{R} \mathbf{l}_{p}^{[c]}$ & $\begin{array}{c}\mathrm{B} 17^{[\mathrm{d}]} \\
\mathrm{E} 10\end{array}$ & $\begin{array}{c}\text { G16 } \\
\text { E1 }\end{array}$ & $\begin{array}{c}\text { G17 } \\
\text { E1 }\end{array}$ & Identification mode \\
\hline a-Pinene & 932 & 1016 & $\mathrm{Tr}$ & - & 0.1 & $\mathrm{RI}, \mathrm{MS}$ \\
\hline Camphene & 942 & 1063 & $\operatorname{Tr}$ & $\operatorname{Tr}$ & 0.2 & $\mathrm{RI}, \mathrm{MS}$ \\
\hline$\delta$-2-carene & 995 & 1130 & 8.6 & 13.1 & 17.0 & $\mathrm{RI}, \mathrm{MS},{ }^{13} \mathrm{C}$ NMR \\
\hline a-Terpinene & 1008 & 1178 & 0.2 & 0.2 & 0.2 & $\mathrm{RI}, \mathrm{MS}$ \\
\hline p-Cymene & 1010 & 1269 & 0.5 & 1.4 & 1.5 & $\mathrm{RI}, \mathrm{MS},{ }^{13} \mathrm{C}$ NMR \\
\hline Limonene* & 1020 & 1199 & 5.9 & 2.4 & 2.5 & $\mathrm{RI}, \mathrm{MS},{ }^{13} \mathrm{C}$ NMR \\
\hline$\beta$-Phellandrene* & 1020 & 1208 & 1.3 & 2.3 & 2.4 & $\mathrm{Rl}, \mathrm{MS},{ }^{13} \mathrm{C}$ NMR \\
\hline (Z)- $\beta$-Ocimene & 1026 & 1230 & 0.4 & 0.3 & 0.4 & $\mathrm{RI}, \mathrm{MS}$ \\
\hline (E)- $\beta$-Ocimene & 1038 & 1247 & 0.2 & 0.1 & 0.2 & $\mathrm{Rl}, \mathrm{MS}$ \\
\hline Terpinolene & 1078 & nd & 0.1 & - & - & $\mathrm{RI}, \mathrm{MS}$ \\
\hline Fenchol & 1098 & 1578 & 0.3 & 0.1 & 0.1 & $\mathrm{RI}, \mathrm{MS}$ \\
\hline cis-p-Menth-2-en-1-ol & 1108 & 1559 & 26.6 & 28.5 & 22.6 & $\mathrm{RI}, \mathrm{MS},{ }^{13} \mathrm{C}$ NMR \\
\hline cis-p-Mentha-2,8-diene-1-ol & 1116 & 1662 & 0.1 & - & 0.1 & $\mathrm{RI}, \mathrm{MS}$ \\
\hline trans- $p$-Menth-2-en-1-ol & 1124 & 1624 & 16.1 & 16.3 & 15.4 & $\mathrm{Rl}, \mathrm{MS},{ }^{13} \mathrm{C}$ NMR \\
\hline Terpineol-4 & 1160 & 1597 & - & 0.3 & 0.2 & $\mathrm{RI}, \mathrm{MS}$ \\
\hline a-Terpineol & 1171 & 1692 & 3.8 & 1.2 & 0.9 & $\mathrm{RI}, \mathrm{MS},{ }^{13} \mathrm{C}$ NMR \\
\hline cis-Piperitol & 1180 & 1674 & 7.1 & 8.5 & 5.4 & $\mathrm{RI}, \mathrm{MS},{ }^{13} \mathrm{C}$ NMR \\
\hline trans-Piperitol & 1191 & 1741 & 8.9 & 8.8 & 8.8 & $\mathrm{RI}, \mathrm{MS},{ }^{13} \mathrm{C}$ NMR \\
\hline Nerol & 1209 & 1795 & 0.1 & 0.1 & - & $\mathrm{RI}, \mathrm{MS}$ \\
\hline Piperitone & 1229 & 1722 & 0.3 & 0.9 & 0.6 & $\mathrm{RI}, \mathrm{MS},{ }^{13} \mathrm{C}$ NMR \\
\hline Geraniol & 1239 & 1842 & 0.1 & 0.3 & 0.3 & $\mathrm{RI}, \mathrm{MS}$ \\
\hline$\beta$-Elemene & 1385 & 1584 & 0.4 & 0.3 & 0.5 & $\mathrm{RI}, \mathrm{MS},{ }^{13} \mathrm{C}$ NMR \\
\hline Y-Muurolene & 1467 & nd & $\operatorname{Tr}$ & - & 0.1 & $\mathrm{RI}, \mathrm{MS}$ \\
\hline Selina-4,11-diene & 1473 & 1667 & $\operatorname{Tr}$ & $\operatorname{Tr}$ & 0.1 & $\mathrm{RI}, \mathrm{MS}$ \\
\hline Germacrene D & 1476 & 1701 & $\operatorname{Tr}$ & $\operatorname{Tr}$ & $\operatorname{Tr}$ & $\mathrm{RI}, \mathrm{MS}$ \\
\hline$\beta$-Selinene & 1479 & 1710 & 0.1 & 0.1 & 0.1 & $\mathrm{RI}, \mathrm{MS}$ \\
\hline$\delta$-Selinene & 1482 & 1685 & $\operatorname{Tr}$ & 0.2 & 0.1 & $\mathrm{RI}, \mathrm{MS}$ \\
\hline 4-epi-Cubebol & 1485 & 1879 & 0.1 & 0.1 & 0.1 & $\mathrm{RI}, \mathrm{MS}$ \\
\hline a-Selinene & 1488 & 1716 & 0.1 & $\operatorname{Tr}$ & 0.1 & $\mathrm{Rl}, \mathrm{MS}$ \\
\hline
\end{tabular}




\begin{tabular}{|c|c|c|c|c|c|c|}
\hline$\gamma$-Cadinene & 1502 & 1751 & 0.3 & 0.2 & 0.3 & $\mathrm{RI}, \mathrm{MS}$ \\
\hline$\delta$-Cadinene & 1510 & 1749 & $\operatorname{Tr}$ & 0.1 & 0.1 & $\mathrm{Rl}, \mathrm{MS}$ \\
\hline$\beta$-Elemol & 1534 & 2074 & 5.6 & 6.4 & 7.4 & $\mathrm{Rl}, \mathrm{MS},{ }^{13} \mathrm{C} \mathrm{NMR}$ \\
\hline Germacrene B & 1549 & 1819 & 0.1 & $\operatorname{Tr}$ & $\operatorname{Tr}$ & $\mathrm{Rl}, \mathrm{MS}$ \\
\hline Spathulenol & 1560 & 2115 & 0.2 & $\operatorname{Tr}$ & $\operatorname{Tr}$ & $\mathrm{Rl}, \mathrm{MS}$ \\
\hline 5-epi,7-epi-a-Eudesmol & 1590 & 2132 & 0.2 & 0.1 & 0.2 & $\mathrm{RI}, \mathrm{MS}$ \\
\hline Selina-6-en-4 $\alpha$-ol\# & 1601 & 2155 & 1.3 & 0.6 & 0.5 & $\mathrm{Rl}, \mathrm{MS},{ }^{13} \mathrm{C}$ NMR \\
\hline Alismol & 1606 & 2245 & 0.1 & 0.1 & 0.1 & $\mathrm{RI}, \mathrm{MS}$ \\
\hline Eremoligenol & 1610 & 2185 & 0.1 & $\operatorname{Tr}$ & $\operatorname{Tr}$ & $\mathrm{RI}, \mathrm{MS}$ \\
\hline$\gamma$-Eudesmol & 1613 & 2159 & 0.1 & 0.1 & 0.2 & $\mathrm{RI}, \mathrm{MS}$ \\
\hline$\tau$-Muurolol & 1619 & 2171 & 0.4 & 0.5 & 0.4 & $\mathrm{Rl}, \mathrm{MS},{ }^{13} \mathrm{C}$ NMR \\
\hline$\tau$-Cadinol & 1621 & 2161 & 0.6 & 0.1 & 0.5 & $\mathrm{Rl}, \mathrm{MS},{ }^{13} \mathrm{C} \mathrm{NMR}$ \\
\hline a-Cadinol & 1625 & 2220 & 0.3 & 0.1 & 0.1 & $\mathrm{RI}, \mathrm{MS}$ \\
\hline$\beta$-Eudesmol* & 1633 & 2220 & 1.7 & 1.8 & 3.7 & $\mathrm{Rl}, \mathrm{MS},{ }^{13} \mathrm{C}$ NMR \\
\hline Valerianol* & 1633 & 2204 & 0.4 & 0.2 & 0.3 & $\mathrm{RI}, \mathrm{MS}$ \\
\hline a-Eudesmol & 1639 & 2211 & 1.2 & 1.1 & 2.3 & $\mathrm{Rl}, \mathrm{MS},{ }^{13} \mathrm{C}$ NMR \\
\hline Intermedeol & 1641 & 2223 & 1.0 & 0.6 & 0.2 & $\mathrm{Rl}, \mathrm{MS},{ }^{13} \mathrm{C} N \mathrm{NMR}$ \\
\hline Juniper camphor & 1677 & 2287 & 1.9 & 0.8 & 0.9 & $\mathrm{Rl}, \mathrm{MS}^{1}{ }^{3} \mathrm{C} \mathrm{NMR}$ \\
\hline Geranyl linalool & 1694 & nd & $\operatorname{Tr}$ & $\operatorname{Tr}$ & $\operatorname{Tr}$ & $\mathrm{RI}, \mathrm{MS}$ \\
\hline Total (\%) & & & 96.9 & 98.1 & 97.0 & \\
\hline
\end{tabular}

[a]: Order of Elution and percentages are given on apolar column (BP-1), excepted those whose names are followed by an asterisk, percentages on polar column (BP-20). ${ }^{[\mathrm{b}]} \mathrm{Rl}_{\mathrm{a}}$ : Retention indices measured on apolar column (BP-1). ${ }^{[\mathrm{cl}} \mathrm{Rl} \mathrm{l}_{\mathrm{p}}$ : Retention indices measured on polar column (BP-20). ${ }^{[d]}$ B17: Béchar 2017, ${ }^{\text {[e] }} \mathrm{G} 16$ : Ghardaïa $2016 \cdot{ }^{13} \mathrm{C}-\mathrm{NMR}=$ compound identified by NMR at least in one oil sample. Tr:

Traces. \#Selina-6-en-4 $\alpha$-ol = Eudesm-6-en-4a-ol.

\section{Oil samples isolated from aerial parts and rhizome of the same plant}

Six C. schoenanthus plants have been harvested in Béchar, aerial parts and rhizomes have been separated manually, and they have been hydrodistilled separately. The twelve oil samples have been analyzed by GC/RI, some by GC/MS and/or by ${ }^{13} \mathrm{C}-\mathrm{NMR}$. The contents of the major components have been reported in Table 2, as mean value (M), standard deviation (SD) and minimum-maximum (min-max).

Table 2: Chemical composition of 6 oil samples isolated from aerial parts and 6 oil samples isolated from rhizomes of the same individual plants.

\begin{tabular}{|c|c|c|c|c|c|c|c|c|c|c|}
\hline \multirow{2}{*}{ Compounds ${ }^{\text {[a] }}$} & \multirow{2}{*}{$\mathbf{R I}_{\mathrm{a}}^{[\mathrm{b}]}$} & \multirow{2}{*}{$\mathbf{R I}_{\mathbf{p}}^{[c]}$} & \multicolumn{4}{|c|}{$A P^{[d]}$} & \multicolumn{4}{|c|}{$\mathbf{R}^{[\mathrm{e}]}$} \\
\hline & & & $M^{[f]}$ & $S D^{[g]}$ & $\operatorname{Min}^{[h]}$ & $\operatorname{Max}^{[i]}$ & $M^{[f]}$ & $S^{[g]}$ & $\operatorname{Min}^{[h]}$ & Maxi \\
\hline$\delta$-2-Carene & 995 & 1130 & 9.0 & 3.7 & 2.1 & 12.6 & 7.6 & 1.4 & 4.8 & 8.5 \\
\hline$p$-Cymene & 1010 & 1269 & 2.1 & 0.4 & 0.3 & 0.7 & 2.6 & 1.1 & 0.4 & 0.8 \\
\hline Limonene* & 1020 & 1199 & 1.0 & 0.3 & 1.6 & 2.5 & 1.0 & 0.3 & 1.6 & 4.1 \\
\hline$\beta$-Phellandrene* & 1020 & 1208 & 0.3 & 0.1 & 0.6 & 1.4 & 0.4 & 0.1 & 0.7 & 1.4 \\
\hline (Z)- $\beta$-Ocimene & 1026 & 1230 & 0.1 & 0.1 & 0.2 & 0.5 & 0.1 & 0.1 & 0.3 & 0.5 \\
\hline cis-p-Menth-2-en-1-ol & 1108 & 1559 & 24.0 & 8.6 & 10.5 & 31.6 & 28.1 & 3.3 & 21.8 & 31.7 \\
\hline trans-p-Menth-2-en-1-ol & 1124 & 1624 & 15.5 & 4.8 & 7.2 & 19.9 & 18.5 & 1.8 & 15.8 & 20.2 \\
\hline$\alpha$-Terpineol & 1171 & 1692 & 1.7 & 0.6 & 1.1 & 2.7 & 2.5 & 1.3 & 1.4 & 3.8 \\
\hline cis-Piperitol & 1180 & 1674 & 7.5 & 3.4 & 3.2 & 12.6 & 8.5 & 1.6 & 5.7 & 10.8 \\
\hline trans-Piperitol & 1191 & 1741 & 9.3 & 2.8 & 4.6 & 12.0 & 11.1 & 1.2 & 9.7 & 12.5 \\
\hline Piperitone & 1229 & 1722 & 0.6 & 0.2 & 0.2 & 0.9 & 0.7 & 0.2 & 0.4 & 0.8 \\
\hline$\beta$-Elemol & 1534 & 2074 & 4.5 & 3.6 & 0.5 & 9.8 & 2.6 & 1.8 & 1.4 & 6.1 \\
\hline Selina-6-en-4 $\alpha$-ol\# & 1601 & 2155 & 2.0 & 1.6 & 0.8 & 5.1 & 1.5 & 0.1 & 1.4 & 1.5 \\
\hline$\beta$-Eudesmol & 1633 & 2220 & 2.1 & 1.6 & 0.3 & 4.0 & 1.2 & 1.1 & 0.4 & 3.3 \\
\hline$\alpha$-Eudesmol & 1639 & 2211 & 1.4 & 1.1 & 0.1 & 3.0 & 0.7 & 0.4 & 0.4 & 1.5 \\
\hline Intermedeol & 1641 & 2223 & 1.5 & 1.0 & 0.7 & 3.3 & 1.2 & 0.2 & 1.0 & 1.6 \\
\hline
\end{tabular}


[a]: Order of elution and percentages are given on apolar column (BP-1), excepted those whose names are followed by an asterisk, percentages on polar column (BP-20). [b] RI Retention indices measured on apolar column (BP-1). ${ }^{[c]} \mathrm{RI}$ : Retention indices measured on polar column (BP-20). ${ }^{[d]} \mathrm{AP}$ : Aerial parts. ${ }^{[\mathrm{le}]} \mathrm{R}: \mathrm{Rhizomes}$. ${ }^{[\mathrm{ff}} \mathrm{M}$ : Mean value. ${ }^{[\mathrm{gl}} \mathrm{SD}$ :

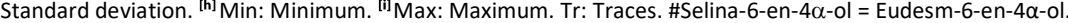

All twelve compositions were dominated by cis-p-menth-2-en-1-ol $(24.0 \% \pm 8.6$ and $28.1 \% \pm 3.3$, respectively) and trans-p-menth-2-en-1-ol $(15.5 \% \pm 4.8$ and $18.5 \% \pm 1.8$, respectively). Other components present at appreciable content were cis- and trans-piperitols, $\beta$-elemol, selina- 6 -en- $4 \alpha-o l$, and juniper camphor. Although slight differences have been observed, they were only quantitative and it could be concluded that aerial parts and rhizome produced similar essential oils.

Variability of 24 oil compositions

Twenty-four plants have been harvested, 10 in Béchar, 14 in Ghardaïa and hydrodistilled. Yields varied from 0.5 to $1.8 \%$. The oil samples have been analyzed by GC/RI, 5 samples by GC/MS and 5 samples by ${ }^{13} \mathrm{C}$ NMR, 3 samples by both techniques. The 24 compositions have been submitted to statistical analysis.

The dendrogram reported below displayed a large group of 20 oil samples with similar composition. For every component with appreciable content mean value, standard deviation as well as minimum and maximum contents have been reported on table 3, which contains also the major components of the last four compositions, classified as "atypical" since they do not constitute a minor group.

Concerning the main group, cis-p-menth-2-en-1-ol $(M=22.6 \% \pm 3.6)$ and trans-p-menth-2-en-1-ol $(M=14.3 \% \pm 1.7)$ were the major components followed by three monoterpenes namely $\delta$-2-carene $(M=9.5 \% \pm 3.1)$, trans-piperitol $(M=8.3 \% \pm 0.9)$, cis-piperitol $(M=7.2 \% \pm 1.8)$ and a sesquiterpene, $\beta$-elemol $(M=7.3 \% \pm 3.1)$. Other oxygenated sesquiterpenes present at appreciable contents were $\beta$-eudesmol, $\alpha$-eudesmol, juniper camphor, selina- 6 en-4a-ol and intermedeol.

Concerning the four, out of 24, atypical oil samples, two of these are from Béchar station, the two others from Ghardaïa station. Compared to the mean compositions of the main group, the two samples from Béchar (B19 E5 and B19 E4) contained more limonene (10.0 and 12.1\% vs. 2.9\% \pm 2.0$)$, aterpineol $(7.4$ and $8.3 \%$ vs. $2.2 \% \pm 1.7)$, and a little bit more intermedeol while $\delta$-2-carene was present at lesser extent $(3.8 \%$ vs. $9.5 \% \pm 3.1)$. The first atypical sample from Ghardaïa (G16 E2) appeared among the richest oil samples in menthenol isomers ( $25.2 \%$ and $18.5 \%$, respectively) while transpiperitol was abundant $(12.8 \%$ vs. $8.3 \% \pm 0.9)$ and in contrast, the content of $\delta$-2-carene was very low $(1.0 \%)$. Lastly, the second oil sample from Ghardaïa (G17 E3) displayed the lowest content of menthenol isomers ( 11.6 and $8.2 \%$, respectively) and it was characterized by its very high content of $\beta$-elemol ( $20.7 \%$ vs. $7.3 \% \pm 3.1)$ and $\beta$-eudesmol (7.2\% vs. $3.0 \% \pm 1.4)$.

Table 3: Mean components of 20 oil samples isolated from the whole plant of $C$. schoenanthus and compositions of four atypical oil samples.

\begin{tabular}{|c|c|c|c|c|c|c|c|c|c|c|}
\hline \multirow{2}{*}{ Compounds ${ }^{[a]}$} & \multirow{2}{*}{$\mathbf{R l}_{\mathbf{a}}^{[\mathrm{b}]}$} & \multirow{2}{*}{$\mathbf{R} \mathbf{l}_{\mathrm{p}}^{[\mathbf{c c}]}$} & \multirow{2}{*}{$\begin{array}{c}M^{[d]} 20 \\
\text { samples }\end{array}$} & \multirow{2}{*}{$\begin{array}{l}S D^{[e]} 20 \\
\text { samples }\end{array}$} & \multirow{2}{*}{$\operatorname{Min}^{[f]}$} & \multirow{2}{*}{$\operatorname{Max}^{[g]}$} & \multicolumn{4}{|c|}{ Atypical } \\
\hline & & & & & & & B19 E5 & B19 E4 & G16 E2 & G17 E3 \\
\hline$\delta$-2-Carene & 995 & 1130 & 9.5 & 3.1 & 5.4 & 17.0 & 3.8 & 3.8 & 1.0 & 3.6 \\
\hline Limonene* & 1020 & 1199 & 2.9 & 2.0 & 1.4 & 7.2 & 10.0 & 12.1 & 0.3 & 3.5 \\
\hline$\beta$-Phellandrene* & 1020 & 1208 & 1.4 & 0.5 & 0.8 & 2.4 & 1.1 & 1.8 & 0.5 & 1.0 \\
\hline (Z)-B-Ocimene & 1026 & 1230 & 0.4 & 0.4 & 0.1 & 1.6 & 0.7 & 0.6 & 0.1 & 0.1 \\
\hline$(E)$ - $\beta$-Ocimene & 1038 & 1247 & 0.2 & 0.2 & 0.1 & 0.7 & 0.3 & 0.3 & $\operatorname{Tr}$ & $\operatorname{Tr}$ \\
\hline cis-p-Menth-2-en-1-ol & 1108 & 1559 & 22.6 & 3.6 & 15.0 & 28.5 & 17.1 & 17.8 & 25.2 & 11.6 \\
\hline trans- $p$-Menth-2-en-1-ol & 1124 & 1624 & 14.3 & 1.7 & 10.8 & 17.0 & 9.6 & 11.4 & 18.5 & 8.2 \\
\hline a-Terpineol & 1171 & 1692 & 2.2 & 1.7 & 0.9 & 5.8 & 7.4 & 8.3 & 1.3 & 2.7 \\
\hline cis-Piperitol & 1180 & 1674 & 7.2 & 1.8 & 3.9 & 11.0 & 7.3 & 5.1 & 8.6 & 3.3 \\
\hline trans-Piperitol & 1191 & 1741 & 8.3 & 0.9 & 6.5 & 10.1 & 5.6 & 6.4 & 12.8 & 5.1 \\
\hline Piperitone & 1229 & 1722 & 0.9 & 0.5 & 0.3 & 2.2 & 0.3 & 0.1 & 1.1 & 0.5 \\
\hline$\beta$-Elemol & 1534 & 2074 & 7.3 & 3.1 & 2.3 & 13.6 & 7.7 & 6.2 & 5.5 & 20.7 \\
\hline Selina-6-en-4 $\alpha$-ol\# & 1601 & 2155 & 1.2 & 0.5 & 0.4 & 2.4 & 0.5 & 0.4 & 1.3 & 1.5 \\
\hline$\beta$-Eudesmol & 1633 & 2220 & 3.0 & 1.4 & 1.2 & 5.7 & 5.1 & 4.6 & 2.5 & 7.2 \\
\hline a-Eudesmol & 1639 & 2211 & 1.7 & 0.8 & 0.6 & 3.8 & 2.2 & 2.2 & 1.3 & 4.0 \\
\hline Intermedeol & 1641 & 2223 & 1.1 & 0.5 & 0.2 & 2.7 & 4.2 & 3.7 & 1.8 & 1.5 \\
\hline Juniper camphor & 1677 & 2287 & 1.5 & 0.7 & 0.5 & 2.8 & 0.5 & 0.3 & 1.8 & 2.5 \\
\hline
\end{tabular}


[a]: Order of elution and percentages are given on apolar column (BP-1), excepted those whose names are followed by an asterisk, percentages on polar column (BP-20). ${ }^{\text {b] }} \mathrm{Rl}$ : Retention indices measured on apolar column (BP-1). ${ }^{[\mathrm{cl}} \mathrm{Rl}$ : Retention indices measured on polar column (BP-20). ${ }^{[\mathrm{d}]}$ M: Mean value. ${ }^{[\mathrm{e}]}$ SD: Standard deviation. ${ }^{[\mathrm{f}]}$ Min: Minimum.

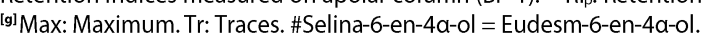

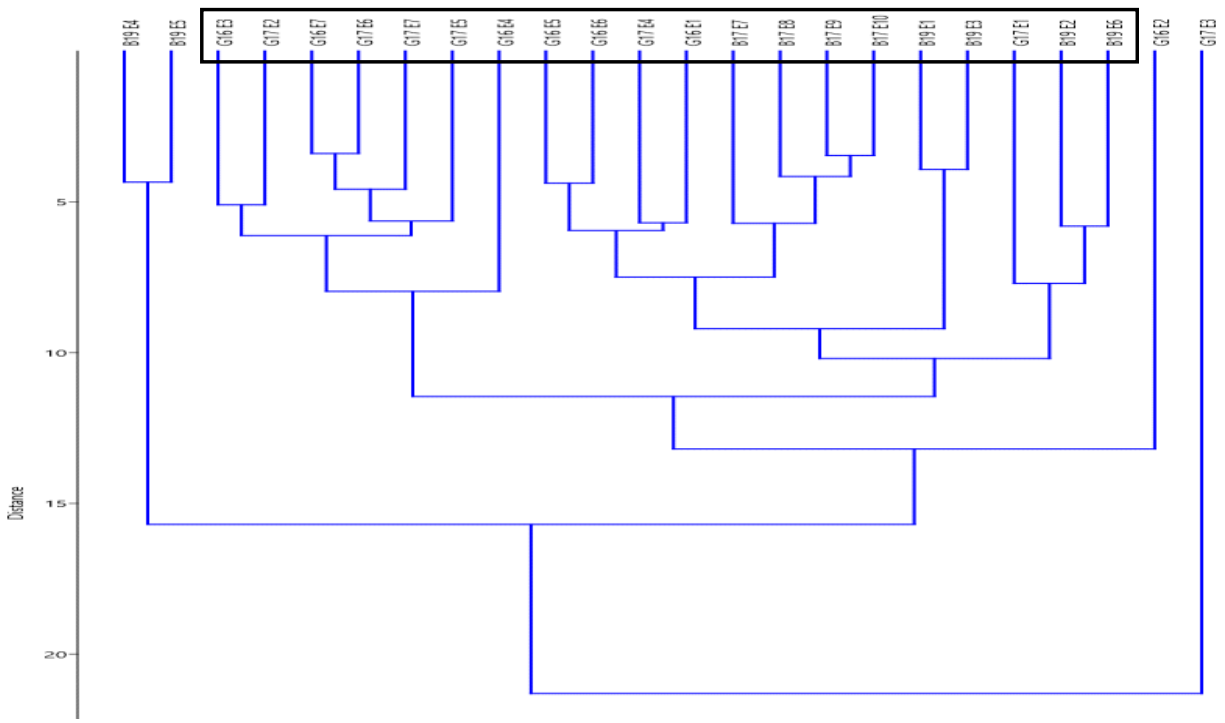

Figure 1. Dendrogram of hierarchical cluster analysis of the $24 \mathrm{C}$. schoenanthus essential oil samples.

\section{Antimicrobial activity of $C$. schoenanthus EO}

Various studies reported on the antimicrobial activity of Algerian C. schoenanthus essential oil. However, results differed from paper to paper: - For instance, zone of inhibition of piperitone-rich (63.3\%) C. schoenanthus essential oil (from Illizi province) against bacterial strains varied from $10.5 \pm$ $0.7 \mathrm{~mm}$ to $21.75 \pm 0.3 \mathrm{~mm}$ and was equal to $12.0 \pm 1.4 \mathrm{~mm}$ for yeast. ${ }^{[31]}$ Piperitone-rich (63.2\% and $55.1 \%$ ) oil samples from Djanet and Tamanrasset showed inhibition zones of $15 \pm 1.2 \mathrm{~mm}$ to $38 \pm 0.7 \mathrm{~mm}$ against various microbial strains; ${ }^{[30]}$

- Another oil sample, whose composition was dominated by guaiol (20.4\%) and cis- $\beta$-terpineol (16.2\%), displayed zones of inhibition of Gram + bacteria in the range of $6.0 \pm 0.0-16.2 \pm 0.3 \mathrm{~mm}$ and Gram - bacteria in the range $7.3 \pm 0.3-13.7 \pm 0.6 \mathrm{~mm} ;{ }^{[32]}$

- A sesquiterpene-rich oil sample (a-eudesmol, 17.9\%; trans-dauca-4(11),7-diene, 10.7\%), from El-Menia was tested against bacterial strains (zone of inhibition $9.00 \pm 0.50-21.67 \pm 0.58 \mathrm{~mm}$ ), filamentous fungi (zone of inhibition $6.50 \pm 0.50-26.33 \pm 0.58 \mathrm{~mm}$ ) and a yeast (zone of inhibition $7.33 \pm 0.58$ $\mathrm{mm}) ;[33]$

- Lastly, essential oils from Béchar and Ghardaïa were checked. They contained various components at appreciable and similar contents, 2-carene, limonene, cis- $p$-menth-2-en-1-ol, trans-p-menth-2-en-1-ol and elemol. Zones of inhibition were measured $(\mathrm{mm})$ against microbial strains: $S$. aureus, $27 \pm$ $0.6 / 22 \pm 0.3 \mathrm{~mm}$; B. subtilis, $24 \pm 1.0 / 20 \pm 0.4 \mathrm{~mm}$; E. coli $16 \pm 0.7 / 19 \pm 1.2 \mathrm{~mm}$; C. albicans $29 \pm 0.2 / 32 \pm 1.3 \mathrm{~mm}$. ${ }^{30]}$

In order to obtain sufficient quantity of essential oil to carry out antimicrobial experiments, various oil samples with similar compositions have been mixed leading group I and group II, whose main components are reported below (Table 4). As observed in oil samples previously investigated, the compositions of both samples were dominated by cis-p-menth-2-en-1-ol (20.2 and 25.0\%, respectively) and trans-p-menth-2-en-1-ol (14.0 and 15.4\%, respectively) while other components ( $\delta$-2-carene, cis-piperitol, trans-piperitol, $\beta$-elemol) accounted for $5-10 \%$ each.

Table 4: Main components of oil samples group I and group II

\begin{tabular}{|c|c|c|c|c|c|}
\hline Compounds (\%) & Group I & Group II & Compounds (\%) & Group I & Group II \\
\hline$\delta$-2-carene & 9.6 & 8.7 & Piperitone & 1.0 & 1.0 \\
\hline p-cymene & 1.4 & 1.2 & $\beta$-elemol & 8.1 & 5.4 \\
\hline$\beta$-Phellandrene* & 1,5 & 1.3 & $\gamma$-Eudesmol & 1.2 & 0.6 \\
\hline cis-p-Menth-2-en-1-ol & 20.2 & 25.0 & $\beta$-Eudesmol & 4.0 & 2.3 \\
\hline trans- $p$-Menth-2-en-1-ol & 14.0 & 15.4 & a-Eudesmol & 1.5 & 1.3 \\
\hline a-Terpineol & 1.2 & 2.9 & Intermedeol & 1.5 & 1.1 \\
\hline cis-Piperitol & 5.7 & 8.0 & Juniper camphor & 1.8 & 1.4 \\
\hline
\end{tabular}


Both oil samples have been checked against various microbial strains, bacteria, yeast and filamentous fungi (Table 5). Concerning bacterial strains, diameters of inhibition zone varied from $8.0 \pm 0.0 \mathrm{~mm}$ to $10.3 \pm 0.6 \mathrm{~mm}$ for Escherichia coli, Klebsiella pneumoniae and Pseudomonas aeruginosa. Staphylococcus aureus was much more sensitive with diameters of inhibition equal to $19.3 \pm 1.2 \mathrm{~mm}$ and $30.7 \pm 0.6 \mathrm{~mm}$, for oil samples Gl and Gll, respectively. The higher activity of essential oil Gll could be highlighted.

Concerning the yeast Candida albicans, a fair activity of both oils was observed, oil Gl being a little bit more active than Gll (24.0 $\pm 3.5 \mathrm{~mm}$ and $19.5 \pm 6.4 \mathrm{~mm}$, respectively). Lastly, concerning filamentous fungi, a fair activity of both oil samples was observed against Aspergillus flavus (13.5 \pm 2.1 $\mathrm{mm}$ and $24.5 \pm 0.7 \mathrm{~mm}$ ) and Fusarium oxysporum (19.0 $\pm 1.4 \mathrm{~mm}$ and $32.5 \pm 0.7 \mathrm{~mm})$. It could be pointed out that zones of inhibition were in the same range than those measured by Aous et al. ${ }^{[30]}$ (essential oils from Béchar and Ghardaïa, respectively) against microbial strains: $S$. aureus: $27 \pm 0.6 / 22 \pm 0.3$ $\mathrm{mm}$; E. coli: $16 \pm 0.7 / 19 \pm 1.2 \mathrm{~mm}$; C. albicans: $29 \pm 0.2 / 32 \pm 1.3 \mathrm{~mm}$.

Table 5: Inhibition zones ( $\mathrm{mm}$ ) of oil collective samples group I and group II against various microbial strains.

\begin{tabular}{|c|c|c|c|c|c|}
\hline \multirow{2}{*}{ Microbial strains } & \multicolumn{2}{|c|}{ Essential oil (15 $\mu \mathrm{L} /$ disc) } & \multicolumn{3}{|c|}{ Positive controls } \\
\hline & Group I & Group II & CIP $(10 \mu \mathrm{g} / \mathrm{disc})$ & NY $(30 \mu \mathrm{g} / \mathrm{disc})$ & FLU $(25 \mu \mathrm{g} / \mathrm{disc})$ \\
\hline \multicolumn{6}{|l|}{ Bacterial strains } \\
\hline Escherichia coli & $10.0 \pm 0.0^{[a]}$ & $9.7 \pm 0.6$ & $34.7 \pm 0.6$ & - & - \\
\hline Klebsiella pneumoniae & $8.0 \pm 0.0$ & $10.3 \pm 0.6$ & $27.7 \pm 0.6$ & - & - \\
\hline Staphylococcus aureus & $19.3 \pm 1.2$ & $30.7 \pm 0.6$ & $30.0 \pm 0.0$ & - & - \\
\hline Pseudomonas aeruginosa & $8.0 \pm 0.0$ & $8.0 \pm 0.0$ & $30.0 \pm 0.0$ & - & - \\
\hline \multicolumn{6}{|l|}{ Yeast } \\
\hline Candida albicans & $24.0 \pm 3.5$ & $19.5 \pm 6.4$ & - & $16.0 \pm 0.0$ & $6.0 \pm 0.0$ \\
\hline \multicolumn{6}{|l|}{ Fungi } \\
\hline Aspergillus flavus & $13.5 \pm 2.1$ & $24.5 \pm 0.7$ & - & $22.3 \pm 0.6$ & $6.0 \pm 0.0$ \\
\hline Fusarium oxysporum & $19.0 \pm 1.4$ & $32.5 \pm 0.7$ & - & $16.0 \pm 1.0$ & $6.0 \pm 0.0$ \\
\hline
\end{tabular}

[a]: Mean values (three experiments) \pm Standard deviation of the growth inhibition zones, in $\mathrm{mm}$, including the disc diameter of $6 \mathrm{~mm}$. CIP: Ciprofloxacin, FLU: Fluconazole. NY: Nystatin were used as positive controls. - : Not tested.

Taking into account these good results, we measured the Minimum Inhibitory Concentrations (MIC) of oil samples Gl and Gll against the most sensitive strains (Table 6). Indeed, MIC values allow a better comparison with antimicrobial activity of selected essential oil reported in literature than diameters of inhibition. Essential oils Gl and Gll displayed more or less similar antimicrobial activity, against one bacterial strain, one yeast and two filamentous fungi, slight differences being observed in some cases. A moderate activity against Aspergillus flavus, Fusarium oxysporum and overall Candida albicans was obtained with both oil samples. A fair activity was observed against Staphylococcus aureus with a MIC value of $0.5 \mu \mathrm{L} / \mathrm{mL}$. The observed MIC value was comparable to that obtained with Algerian carvacrol-rich oils from Thymus fontanesii (MIC $=0.3 \mathrm{mg} / \mathrm{mL})^{[37]} \mathrm{and}$ Thymus ciliatus $(\mathrm{MIC}=0.9 \mathrm{mg} / \mathrm{mL}) \cdot{ }^{[38]}$ In the absence of phenol derivatives, it could be assumed that this fair activity could be attributed to the high amount of various alcohols bearing the p-menthane skeleton (cis-p-menth-2-en-1-ol, trans-p-menth-2-en-1-ol, cis- and trans-piperitols). However, oxygenated sesquiterpenes such as $\beta$-elemol and $\beta$-eudesmol could also contribute to this activity in synergism with major components.

Table 6: Minimum Inhibitory Concentrations (MICs) of C. schoenanthus of Gl and Gll collective samples against microbial strains.

\begin{tabular}{lcc}
\hline \multirow{2}{*}{ Microbial strains } & \multicolumn{2}{c}{ MIC $(\boldsymbol{\mu L} / \mathbf{m L})$} \\
\cline { 2 - 3 } & Sample Group I & Sample Group II \\
\hline Staphylococcus aureus & 0.5 & 0.5 \\
Candida albicans & 4.0 & 3.0 \\
Aspergillus flavus & 2.0 & 2.0 \\
Fusarium oxysporum & 2.0 & 2.0 \\
\hline
\end{tabular}

Antioxidant activity of C. schoenanthus EO

The antioxidant ability of essential oils is generally associated with the presence of phenolic compounds such as thymol and carvacrol. Nevertheless, it has also been shown that essential oils containing mainly oxygenated monoterpenes displayed an appreciable antioxidant activity. 
Moreover, the antioxidant activity of the monoterpene hydrocarbons fraction has been demonstrated. ${ }^{[39]}$ Concerning C. schoenanthus essential oils, a low antioxidant activity has been observed [Aous et al, 2019]. ${ }^{[30]}$ However, $\mathrm{I}_{50}$ value was not measured.

Therefore, the in vitro antioxidant activity of $C$. schoenanthus essential oils against DPPH free radical scavenging has been assessed and compared to that of ascorbic acid. The inhibitory potential of the essential oils against DPPH depended gradually increased, between $3.83 \pm 0.16$ to $93.78 \pm 0.11 \%$, with an increasing concentration of essential oil $\left(0.125\right.$ to $256 \mathrm{mg} / \mathrm{mL}$ ) (Figure 2). Both oil samples exhibited similar antioxidant power, although $\mathrm{IC}_{50}$ value varied from sample to sample $\left(I_{50}=10.66 \pm 0.1\right.$ and $16.30 \pm 0.3 \mathrm{mg} / \mathrm{mL}$, Table 7). Whatever the sample, the antioxidant potential of $C$. schoenanthus essential oil was significantly lower than that of ascorbic acid employed as positive control $\left(\mathrm{IC}_{50}=0.02 \pm 0.001 \mathrm{mg} / \mathrm{mL}\right)$.

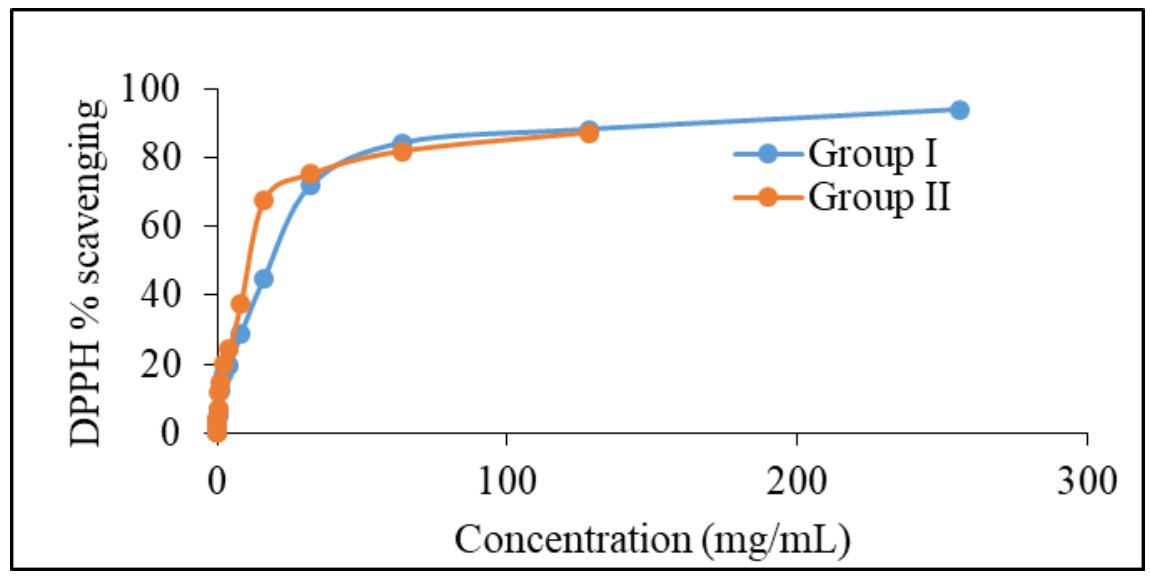

Figure 2. Scavenging effect on $\mathrm{DPPH}^{\circ}$ of $C$. schoenanthus oil samples.

Table 7: Antioxidant test measured by DPPH radical scavenging capacity: $I C_{50}$ values of $C$. schoenanthus essential oil.

\begin{tabular}{cccc}
\hline \multirow{2}{*}{ I $_{50}(\mathrm{mg} / \mathrm{mL})$} & Group I & Group II & Ascorbic acid \\
\cline { 2 - 4 } & $16.30 \pm 0.3$ & $10.66 \pm 0.1$ & $0.02 \pm 0.001$ \\
\hline
\end{tabular}

\section{Conclusions}

Cymbopogon schoenanthus is an aromatic plant whose aerial parts and rhizome produced an essential oil with pleasant odor. Various compositions of the essential oil have been reported depending of the countries were the plant grows wild. Such a chemical variability has been observed in Algeria where a composition dominated by cis- and trans-p-menth-2-en-1-ols occurred, not found in other countries. We demonstrated that in two locations of harvest, Béchar and Ghardaïa (Central Algeria), the essential oil composition is homogenous for 20 oil samples out of 24 , and therefore, this is a positive point for an eventual production and domestication of $C$. schoenanthus essential oil. Otherwise, a fair antimicrobial activity was measured against Staphylococcus aureus strain, while the antioxidant potential was low.

\section{Experimental Section}

\section{Plant Material and Oil Distillation}

Whole plant (WP), rhizome (R), aerial parts (AP: stems and leaves) from 30 individual plants $C$. schoenanthus were collected in two locations of Algeria (Figure 3), sixteen plants were sampled at Béchar province (July 2017: B17 AP E1 - 6; B17 RE1 - 6, B17 WP E7 - 10 and in April 2019: B19 WP E1 - 6) and fourteen plants were sampled at Ghardaïa province (seven in April 2016: G16 WP E1 - 7 and seven in January 2017: G17 WP E1 - 7). Identification of the plants was performed by Dr. F. Hassani (University of Tlemcen, Algeria). A voucher specimen has been deposited with the Laboratory of Natural Products (Department of Biology, University of Tlemcen) under the accession No. P. 111. Dry plants $(60-120 \mathrm{~g})$ were submitted to hydrodistillation for 2 h. Yields have been calculated from dry material $(w / w)$. 


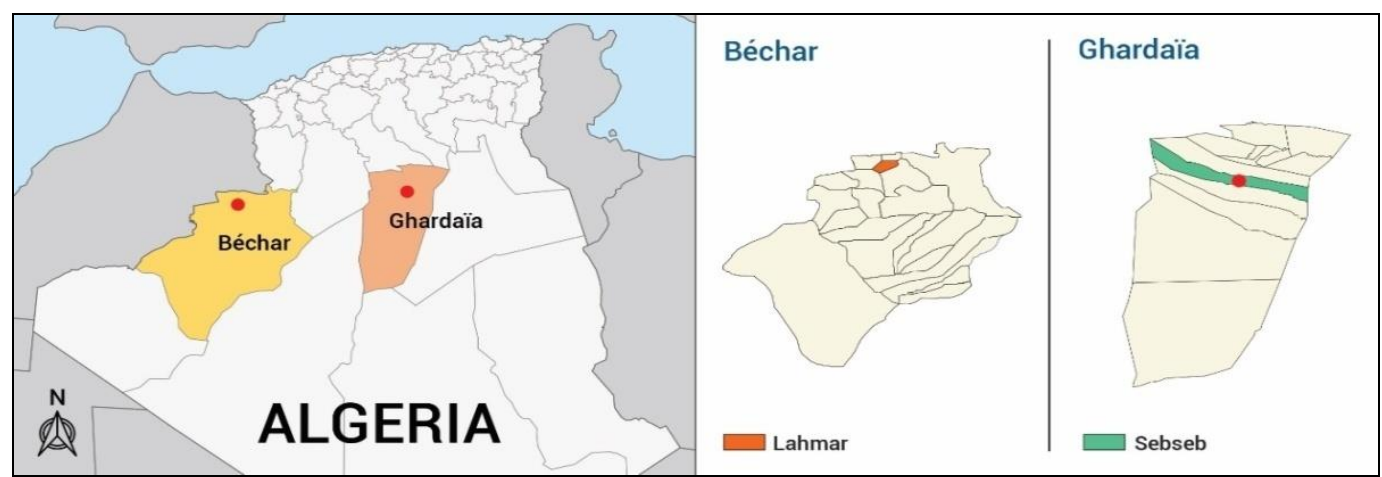

Figure 3. Sampling locations of Cymbopogon schoenanthus from Algeria.

\section{Analytical GC}

GC analyzes were performed on a Perkin-Elmer Clarus 500 gas chromatograph (FID) equipped two fused silica capillary columns $(50 \mathrm{~m} \times 0.22 \mathrm{~mm}$, $0.25 \mu \mathrm{m}$ film thickness), BP-1 (polydimethyl siloxane) and BP-20 (polyethylene glycol). The oven temperature was programmed from $60^{\circ} \mathrm{C}$ to $220^{\circ} \mathrm{C}$ at $2{ }^{\circ} \mathrm{C} / \mathrm{min}$ and then held isothermal at $220^{\circ} \mathrm{C}$ for $20 \mathrm{~min}$, injector temperature: $250^{\circ} \mathrm{C}$; detector temperature: $250^{\circ} \mathrm{C}$; carrier gas: hydrogen $(0.8 \mathrm{ml} / \mathrm{min})$; split: $1 / 60$. The relative proportions of the oil constituents were expressed as percentages obtained by peak area normalization, without using correcting factors. Retention indices (RI) were determined relative to the retention times of a series of $n$-alkanes with linear interpolation (“Target Compounds" software from Perkin-Elmer).

\section{GC/MS Analysis}

The essential oils were analyzed with a Perkin-Elmer TurboMass detector (quadrupole), directly coupled to a Perkin-Elmer Autosystem XL, equipped with a fused-silica capillary column $(50 \mathrm{~m} \times 0.22 \mathrm{~mm}$ i.d., film thickness $0.25 \mu \mathrm{m})$, BP-1(dimethylpolysiloxane). Carrier gas, helium at 0.8 $\mathrm{mL} / \mathrm{min}$; split, $1 / 60$; injection volume, $0.5 \mu \mathrm{L}$; injector temperature, $250^{\circ} \mathrm{C}$; oven temperature programmed from $60^{\circ} \mathrm{C}$ to $220^{\circ} \mathrm{C}$ at $2{ }^{\circ} \mathrm{C} / \mathrm{min}$ and then held isothermal ( $20 \mathrm{~min}$ ); lon source temperature, $250^{\circ} \mathrm{C}$; energy ionisation, $70 \mathrm{eV}$; electron ionisation mass spectra were acquired over the mass range 40-400 Da.

\section{${ }^{13}$ C-NMRAnalysis}

${ }^{13} \mathrm{C}$ NMR analyzes were performed on a Bruker AVANCE 400 Fourier Transform spectrometer operating at $100.623 \mathrm{MHz}$ for ${ }^{13} \mathrm{C}$, equipped with a 5 $\mathrm{mm}$ probe, in deuterated chloroform $\left(\mathrm{CDCl}_{3}\right)$, with all shifts referred to internal tetramethylsilane (TMS). ${ }^{13} \mathrm{CNMR}$ spectra were recorded with the following parameters: pulse width (PW), $4 \mu \mathrm{s}$ (flip angle $45^{\circ}$ ); acquisition time, $2.73 \mathrm{~s}$ for $128 \mathrm{~K}$ data table with a spectral width (SW) of $220000 \mathrm{~Hz}(220$ ppm); CPD mode decoupling; digital resolution $0.183 \mathrm{~Hz} / \mathrm{pt}$. The number of accumulated scans ranged $2000-3000$ for each sample (around $40 \mathrm{mg}$ of oil in $0.5 \mathrm{~mL}$ of $\left.\mathrm{CDCl}_{3}\right)$. Exponential line broadening multiplication $(1.0 \mathrm{~Hz})$ of the free induction decay was applied before Fourier transformation.

\section{Identification of Components}

Identification of the components was based: i) on comparison of their GC retention indices (Rls) on polar and apolar columns, determined relative to the retention times of a series of n-alkanes with linear interpolation ('Target Compounds' software of Perkin-Elmer), with those of authentic compounds and with reference data ${ }^{[40]}$ on computer matching against commercial mass spectral libraries ${ }^{[40-43]}$ and (iii) on comparison of the signals in the ${ }^{13} \mathrm{C}$-NMR spectra of essential oils with those of reference spectra compiled in the laboratory spectral library, with the help of a laboratory-made software. ${ }^{[34-36]}$ In the investigated samples, individual components were identified by NMR at contents as low as $0.3-0.4 \%$.

${ }^{13} \mathrm{C}-\mathrm{NMR}(\delta, \mathrm{ppm})$ of cis- and trans-p-menth-2-en-1-ol. Cis isomer: $133.56(\mathrm{CH}) ; 133.23(\mathrm{CH}) ; 67.48(\mathrm{C}) ; 42.12(\mathrm{CH}) ; 37.28\left(\mathrm{CH}_{2}\right) ; 31.74(\mathrm{CH}) ; 29.65$ $\left(\mathrm{CH}_{3}\right) ; 21.64\left(\mathrm{CH}_{2}\right) ; 19.66\left(\mathrm{CH}_{3}\right) ; 19.31\left(\mathrm{CH}_{3}\right)$, Trans isomer: $134.68(\mathrm{CH}) ; 131.19(\mathrm{CH}) ; 69.60(\mathrm{C}) ; 41.71(\mathrm{CH}) ; 38.03\left(\mathrm{CH}_{2}\right) ; 31.77\left(\mathrm{CH}_{)} ; 28.49\left(\mathrm{CH}_{3}\right) ; 23.59\left(\mathrm{CH}_{2}\right)\right.$; $19.72\left(\mathrm{CH}_{3}\right) ; 19.39\left(\mathrm{CH}_{3}\right) .{ }^{13} \mathrm{C}-\mathrm{NMR}$ values fitted perfectly with those reported. ${ }^{[44]}$

\section{Data Analysis}

Principal Components Analysis (PCA) and Hierarchical clustering (Ward's method) were performed by Xlstat (Adinsoft, France). ${ }^{[4]]}$

\section{Antimicrobial Activity of the Essential Oil}

Microbial Strains 
Antimicrobial activity of the essential oil (collective sample) were evaluated against one Gram positive bacteria (Staphylococcus aureus ATCC 6538 ) and three Gram negative bacteria (Escherichia coli ATCC 8739, Klebsiella pneumoniae ATCC 700603 and Pseudomonas aeruginosa ATCC 27853), one yeast (Candida albicans ATCC 10231) and two filamentous fungi (Fusarium oxysporum MNHN 963917 and Aspergillus flavus MNHN 994294).

Screening of Antimicrobial Activity

The agar diffusion method was used for the determination of antimicrobial activity of the EOs. ${ }^{[45]}$ Briefly, a suspension of the tested microorganisms ( $1 \mathrm{~mL}$ of a suspension at $10^{6}$ cells $/ \mathrm{mL}$ for bacteria and yeast, $10^{7}$ cells $/ \mathrm{mL}$ for S. aureus and $10^{4} \mathrm{spores} / \mathrm{mL}$ for filamentous fungi) was spread on the solid media plates, using Mueller-Hinton agar for bacteria, Sabouraud dextrose for yeast and PDA for filamentous fungi. Filter paper discs ( $6 \mathrm{~mm}$ in diameter) were impregnated with $15 \mu \mathrm{L}$ of the oil and $5 \mu \mathrm{L}$ of DMSO and placed on the surface of inoculated plates. The activity was determined by measuring the inhibitory zone diameter in $\mathrm{mm}$ after incubation for $24 \mathrm{~h}$ at $37^{\circ} \mathrm{C}$ for bacteria, $24-48 \mathrm{~h}$ at $30^{\circ} \mathrm{C}$ for yeast and 5 days at $25^{\circ} \mathrm{C}$ for filamentous fungi. Fluconazole (FLU $25 \mu \mathrm{g} / \mathrm{disc}$ ), nystatin (NY $30 \mu \mathrm{g} / \mathrm{disc}$ ) were used as reference antifungal against yeast and filamentous fungi and ciprofloxacin (CIP $10 \mu \mathrm{g} / \mathrm{disc}$ ) was used as positive controls against bacteria. DMSO was used as negative control. Each test was performed in triplicate.

Determination of minimum inhibitory concentration (MIC)

The MIC was defined as the lowest concentration of tested sample that resulted in complete inhibition of visible growth. Dilutions of oil were made in culture medium over the concentration range $0.25-4 \mu \mathrm{L} / \mathrm{mL} .1 \mathrm{~mL}$ of standardized suspension was added. Inoculated plates were incubated at $37^{\circ} \mathrm{C}$ for $24 \mathrm{~h}$ for bacteria, $24-48 \mathrm{~h}$ at $37^{\circ} \mathrm{C}$ for yeast and 5 days at $25^{\circ} \mathrm{C}$ for filamentous fungi. MICs were determined as the minimum concentration with no visible growth. Each test was performed in duplicate or in triplicate.

\section{DPPH Radical Scavenging Activity}

The antioxidant activity was measured on a sample of essential oil (Collective sample).The antioxidant activity of $C$. schoenanthus EO was measured on the basis to scavenge of the 2.2-diphenyl-1-picrylhydrazil (DPPH) free radical, according to the experimental protocol described by Kouame et al. ${ }^{[46]} \mathrm{A}$ volume of $2.5 \mathrm{~mL}$ with various concentrations $(256,128,64,32,16,8,4,2,1,0.5,0.25,0.125,0.0625,0.03125 \mathrm{and} 0.015625 \mathrm{mg} / \mathrm{mL}$ ) of the EO in absolute ethanol were added to $1 \mathrm{~mL}$ of an ethanolic solution of DPPH at $0.03 \mathrm{mg} / \mathrm{mL}$. For each concentration, a blank was prepared. In parallel, a negative control is prepared by mixing $2.5 \mathrm{~mL}$ of absolute ethanol with $1 \mathrm{~mL}$ of ethanolic solution of DPPH: After incubation in the dark for 30 min at room temperature, the absorbance was measured against a blank at $517 \mathrm{~nm}$. The activity of the EO was compared to ascorbic acid as a positive control. DPPH free radical scavenging activity in percentage (\%) was calculated using the following formula:

$$
\mathrm{DPPH} \text { - scavenging activity }(\%)=[(\text { Acontrol- Asample) } / \text { Acontrol }] \text { X100 }
$$

Acontrol is the absorbance of the negative control; Asample is the absorbance of the tested sample.

The concentration of the EO required for the $50 \%$ reduction in the initial concentration of DPPH $\left(\mathrm{IC}_{50}\right)$ was calculated from the graph plotted of percentage inhibition against essential oil concentrations. Each test was performed in triplicate.

\section{Acknowledgements}

This work was conducted within the cooperative program between the Universities of Abou Bekr Belkaïd (Tlemcen) and Pascal Paoli (Corsica). C. Bekhechi thanks University of Corsica for a research grant as associate professor (September 2018). C. Bekhechi also acknowledges Prof. N.A. MokhtariSoulimane (Dean, Faculty of Life Sciences, Tlemcen) for attribution of a travel grant (September 2019) and Dr F. Hassani, University of Tlemcen, for his help on plant identification.

\section{Author Contribution Statement}

C.B. conceived and designed the experiments; C.E.W.M., I.A.E.H. and F.H. performed sampling, extractions and identification of the plant; M.P. performed GC, MS and NMR experiments; M.G. performed statistical analyses; J.C., C.B. and F.T. contributed to the preparation of the manuscript.

\section{References}


[1] O. Avoseh, O. Oyedeji, P. Rungqu, B. Nkeh-Chungag, A. Oyedeji, 'Cymbopogon Species; Ethnopharmacology, Phytochemistry and the Pharmacological Importance', Molecules, 2015, 20, 7438-7453.

[2] P. Ozenda, 'Flore et végétation du Sahara', 3ème édition, CNRS, Paris, 1991.

[3] P. Quézel, S. Santa, 'Nouvelle flore de l'Algérie et des régions désertiques méridionales', CNRS Paris, France, 1963.

[4] A. Sahki, R. Sahki, 'Le Hoggar, promenade botanique', Éd. Ésope, 2004.

[5] H.I. Heiba, A.M. Rizk, 'Constituents of Cymbopogon species', Quatar Univ. Sci. Bull. 1986, 6, 53-75.

[6] V. Hammiche, K. Maiza, 'Traditional medicine in Central Sahara: Pharmacopoeia of Tassili N'ajjer', J. Ethnopharmacol. 2006, 105, 358-367.

[7] L.M. Katiki, A.C.S. Chagas, H.R. Bizzo, J.F.S. Ferreira, A.F.T. Amarante, 'Anthelmintic activity of Cymbopogon martinii, Cymbopogon schoenanthus and Mentha piperita essential oils evaluated in four different in vitro tests', Vet. Parasitol. 2011, 183, 103-108.

[8] A.K. Shahi, A. Tava, 'Essential oil composition of three Cymbopogon species of Indian Thar Desert', J. Essent. Oil Res. 1993, 5, 639-643.

[9] H.C. Andola, A. Maithani, 'Essential Oil Profile of Wild and Cultivated Accession of Cymbopogon schoenanthus (L.) from Uttarakhand Region', Med. Chem. 2018, 8, 008009. doi: 10.4172/2161-0444.10489.

[10] G.M. Hashim, S.B. Almasaudi, E. Azhar, S.K. Al Jaouni, S. Harakeh, 'Biological Activity of Cymbopogon schoenanthus Essential Oil', Saudi J. Biol. Sci. 2016, doi: http://dx.doi.org/10.1016/j.sjbs.2016.06.001.

[11] M. Golestaneh Talaei, Z. Mousavi, M. Jahandideh, 'Anti-Inflammatory Activity of Cymbopogon schoenanthus Essential Oil in Animal Models', Res. J. Pharmacogn. 2019, 6, 61-68.

[12] G. Nonviho, V.D. Wotto, J.P. Noudogbessi, F. Avlessi, M. Akogbeto, D.C.K. Sohounhloué, 'Insecticidal activities of essential oils extracted from three species of Poaceae on Anopheles gambiae ssp. major vector of malaria', Scient. Study Res. Chem. Chem. Engineer. Biotechnol. Food Ind. 2010, 11, 411-420.

[13] G.A. Alitonou, F. Avlessi, F. Tchobo, J.P. Noudogbessi, A. Tonouhewa, B. Yehouenou, C. Menut, D.K. Sohounhloue, ‘Chemical composition and biological activities of essential oils from the leaves of Cymbopogon giganteus Chiov. and Cymbopogon schoenanthus (L.) Spreng (Poaceae) from Benin', Int. J. Biol. Chem. Sci. 2012, 6, 18191827.

[14] A.D. Bossou, E. Ahoussia, E. Ruysbergh, A. Adams, G. Smagghe, N. De Kimpe, F. Avlessi, D.C.K. Sohounhloue, S. Mangelinckx, ‘Characterization of volatile compounds from three Cymbopogon species and Eucalyptus citriodora from Benin and their insecticidal activities against Tribolium castaneum', Indus. Crops Prod. $2015,76,306-317$.

[15] S. Kpoviessi, J. Bero, P. Agbani, F. Gbaguidi, B. Kpadonou-Kpoviessi, B. Sinsin, G. Accrombessi, M. Frédérich, M. Moudachirou, J. Quetin-Leclercq, ‘Chemical composition, cytotoxicity and in vitro antitrypanosomal and antiplasmodial activity of the essential oils of four Cymbopogon species from Benin', J. Ethnopharmacol. 2014, 151, 652659.

[16] D. Kpadonou, F. Allanto, B. Kpadonou-Kpoviessi, P. Agbani, F. Gbaguidi, L. Baba-Moussa, J. Gbenou, M. Moudachirou, S. Kpoviessi, 'Relations entre composition chimique, activité antioxydante et toxicité des huiles essentielles de deux espèces de Cymbopogon acclimatées au Bénin', Int. J. Biol. Chem. Sci. 2019, 13, 1201-1209.

[17] K. Koba, K. Sanda, C. Raynaud, Y.A. Nenonene, J. Millet, J.P. Chaumont, 'Activités antimicrobiennes d'huiles essentielles de trois Cymbopogon sp. Africains vis-à-vis de germes pathogènes d'animaux de compagnie', Ann. Méd. Vét. 2004, 148, 202-206.

[18] G.K. Ketoh, H.K. Koumaglo, I.A. Glitho, 'Inhibition of Callosobruchus maculatus (F.) (Coleoptera: Bruchidae) development with essential oil extracted from Cymbopogon schoenanthus L. Spreng. (Poaceae), and the wasp Dinarmus basalis (Rondani) (Hymenoptera: Pteromalidae)', J. Stored Prod. Res. 2005, $41,363-371$.

[19] K.A. Gbogbo, K. Batawila, K. Anani, M. Prince-David, M. Gbeassor, P. Bouchet, K. Akpagana, 'Activité antifongique des huiles essentielles de Ocimum basilicum L. (Lamiaceae) et Cymbopogon schoenanthus (L.) Spreng. (Poaceae) sur des micromycetes influençant la germination du Maïs et du Niébé', Acta Bot. Gallica 2006, 153, 115-124.

[20] N.A. Nadio, K. Koba, W. Poutouli, P. Akantetou, B. Laba, M.E. Bokobana, C. Raynaud, K. Sanda, 'Activités insecticides de l'huile essentielle de Cymbopogon schoenanthus (L.) Spreng. sur les larves de stade II de Dysdercus voelkeri Schmidt (Heteroptera:Pyrrhocoridae)', J. Soc. Ouest-Afr. Chim. 2013, 35, 8-14.

[21] E.M. Bokobana, K. Koba, W.P. Poutouli, P. K. Akantetou, N. A. Nadio, B. Laba, P. Tozoou, C. Raynaud, K. Sanda, 'Evaluation du potentiel insecticide et répulsive de I'huile essentielle de Cymbopogon schoenanthus (L) Spreng. sur Aphis gossypii Glover (Homoptera: Aphididae), ravageur du cotonnier du Togo', Rev. Cames Sci. Vie Terre Agro. 2014, 2, 48-55.

[22] K.B.J.N. Gbenyedji, W.S. Nyamador, B.D. Kasseney, C.H.R. Nebie, K.G. Ketoh, K.K.E. Anani, A.I. Glitho, 'The use of two new formulations of Ocimum canum Sims and Cymbopogon schoenanthus L. in the control of Amitermes evuncifer Silvestri (Termitidae: Termitinae) in Togo', Int. J. Nat. Sci. Res. 2014, 2, 195-205.

[23] Y. Onadja, A. Ouedraogo, A.D. Samate, 'Chemical composition and physical characteristics of the essential oil of Cymbopogon schoenanthus Spreng of Burkina Faso', J. Applied Sci. 2007, 7, 503-506.

[24] S. Yagi, R. Babiker, T. Tzanova, H. Schohn, 'Chemical composition, antiproliferative, antioxidant and antibacterial activities of essential oils from aromatic plants growing in Sudan', Asian Pacific J. Tropical Med. 2016, 9, 763-770.

[25] I. Pavlović, E. Omar, M. Drobac, M. Radenković, S. Branković, N. Kovačević, 'Chemical composition and spasmolytic activity of Cymbopogon schoenanthus (L.) Spreng. (Poaceae) essential oil from Sudan', Arch. Biol. Sci. 2017, 69, 409-415.

[26] A. Khadri, M.L.M. Serralheiro, J.M.F. Nogueira, M. Neffati, S. Smiti, M.E.M. Araujo, 'Antioxidant and antiacetylcholinesterase activities of essential oils from Cymbopogon schoenanthus L. Spreng. Determination of chemical composition by GC-mass spectrometry and ${ }^{13} \mathrm{C} \mathrm{NMR}^{\prime}$, Food Chem. 2008, $109,630-637$.

[27] E.M.B.D. de Sousa, A.P. Costa Câmara, W. A. Costa, A.C.J. Costa, H.N.M. Oliveira, E. Lopes Galvão, M.M Ortiz Marques, 'Evaluation of the Extraction Process of the Essential Oil from Cymbopogon schoenanthus with Pressurized Carbon Dioxide', Braz. Arch. Biol. Technol. 2005, 48, $231-236$.

[28] Z. Bouchikhi Tani, M.A. Khelil, M. Bendahou, M. Mestari, 'Action des huiles essentielles de trois plantes aromatiques de l'Ouest algérien sur la mite Tineola bisselliella', Rev. Ivoir. Sci. Technol. 2009, 14, 175-186.

[29] D. Hadef, M. Saidi, M. Yousfi, Y. Moussaoui, 'GC/MS Analysis of essential oils of Cymbopogon schoenanthus and Origanum majorana L. grown in Eastern Algeria', Asian J. Chem. 2015, 27, 3575-3578. 
[30] W. Aous, O. Benchabane, T. Outaleb, M. Hazzit, F. Mouhouche, A. Yekkour, A. Baaliouamer, 'Essential oils of Cymbopogon schoenanthus (L.) Spreng. from Algerian Sahara: chemical variability, antioxidant, antimicrobial and insecticidal properties', J. Essent. Oil Res. 2019, DOI: 10.1080/10412905.2019.1612790.

[31] N. Hellali, M.H. Mahammed, F. Ramdane, A. Talli, 'Antimicrobial and antioxidant activities of Cymbopogon schoenanthus (L.) Spreng. essential oil, growing in IlliziAlgeria', J. Med. Plants Res. 2016, 10, 188-194.

[32] M. Kadri, N. Salhi, A. Yahia, K. Amiar, H. Gnabzia, 'Chemical composition, antioxidant and antimicrobial activities from extracts of Cymbopogon schoenanthus L. (Spreng) of Algeria', Int. J. Biosci. 2017, 10, 318-326.

[33] F.-Z. Bellik, F. Benkaci-Ali, Z. Alsafra, G. Eppe, S. Tata, N. Sabaou, R. Zidani, 'Chemical composition, kinetic study and antimicrobial activity of essential oils from Cymbopogon schoenanthus L. Spreng extracted by conventional and microwave-assisted techniques using cryogenic grinding', Indus. Crops Prod. $2019,139,111505$.

[34] F. Tomi, P. Bradesi, A. Bighelli, J. Casanova, 'Computer-aided identification of individual components of essential oils using Carbon-13 NMR spectroscopy', J. Magn. Reson. Anal. 1995, 1, 25-34.

[35] F. Tomi, J. Casanova, ${ }^{13} \mathrm{C}-\mathrm{NMR}$ as a tool for identification of individual components of essential oils from Labiatae. A review', In "The Labiatae: Advances in Production, Biotechnology and Utilization" Eds: Cervelli C, Ruffoni B, Dalla Guda C. Acta Hortic. 2006, 723, 185-192.

[36] Z.A. Ouattara, J.B. Boti, A.C. Ahibo, S. Sutour, J. Casanova, F. Tomi, A. Bighelli, 'The key role of ${ }^{13} \mathrm{C}$ NMR analysis in the identification of individual components of Polyalthia longifolia leaf oil', Flavour Fragr J. 2014,29, 371-379.

[37] C. Bekhechi, F. Atik Bekkara, D.E. Abdelouahid, F. Tomi, J. Casanova, 'Composition and antibacterial activity of the essential oil of Thymus fontanesii Boiss. et Reut. from Algeria', J Essent. Oil Res. 2007, 19, 594-596.

[38] L. Bousmaha-Marroki, F. Atik-Bekkara, F. Tomi, J. Casanova, 'Chemical Composition and Antibacterial Activity of the Essential Oil of Thymus ciliatus (Desf.) Benth. ssp. eu-ciliatus Maire from Algeria', J. Essent. Oil Res. 2007, 19, 490-493.

[39] M.G. Miguel, 'Antioxidant activity of medicinal and aromatic plants. A review', Flavour Fragr. J. 2010, 25, 291-312.

[40] W.A. König, D. H. Hochmuth, D. Joulain,'Terpenoids and Related Constituents of Essential Oils', Library of MassFinder 2.1', Institute of Organic Chemistry, Hamburg, 2001.

[41] National Institute of Standards and Technology, 'PC Version 1.7 of the NIST/EPA/NIH Mass Spectral Library', Perkin-Elmer Corporation, Norwalk, CT, USA, 1999.

[42] National Institute of Standards and Technology, 'PC version of the mass spectral library', Norwalk, CT, USA, 2014.

[43] R.P. Adams, 'Identification of Essential Oils Components by Gas Chromatography/ Mass Spectroscopy', Carol Stream, Allured (4th edition), 2007.

[44] T. Kashiwagi, T. Nakashima, S.-I. Tebayashi, C.-S. Kim, 'Determination of the Absolute Configuration of Quercivorol, (1S,4R)-p-Menth-2-en-1-ol, an Aggregation Pheromone of the Ambrosia Beetle Platypus quercivorus (Coleoptera: Platypodidae)', Biosci. Biotechnol. Biochem. 2006, 70, $2544-2546$.

[45] NCCLS (National Committee for Clinical Laboratory Standards), 'Performance standards for antimicrobial susceptibility testing: eleventh informational supplement', NCCLS document M100-S11. USA: Villanova, 21(1), 2001.

[46] C. Kouame, Z.A. Ouattara, M.K. Konan, C.KC. N'Gaman-Kouassi, F. Tomi, J.A. Mamyrbekova-Bekro, Y-A. Bekro, 'Quantitative organic composition and antioxidant potential oh the essential oil from Origanum syriacum L. (Lamiaceae) acclimated in Côte d'Ivoire', Int.J. Curr. Res. 2017, 10, 73602-73605.

[47] P. Legendre, L. Legendre, 'Numerical Ecology', 2nd edn., Elsevier Science, Amsterdam, 1998. 


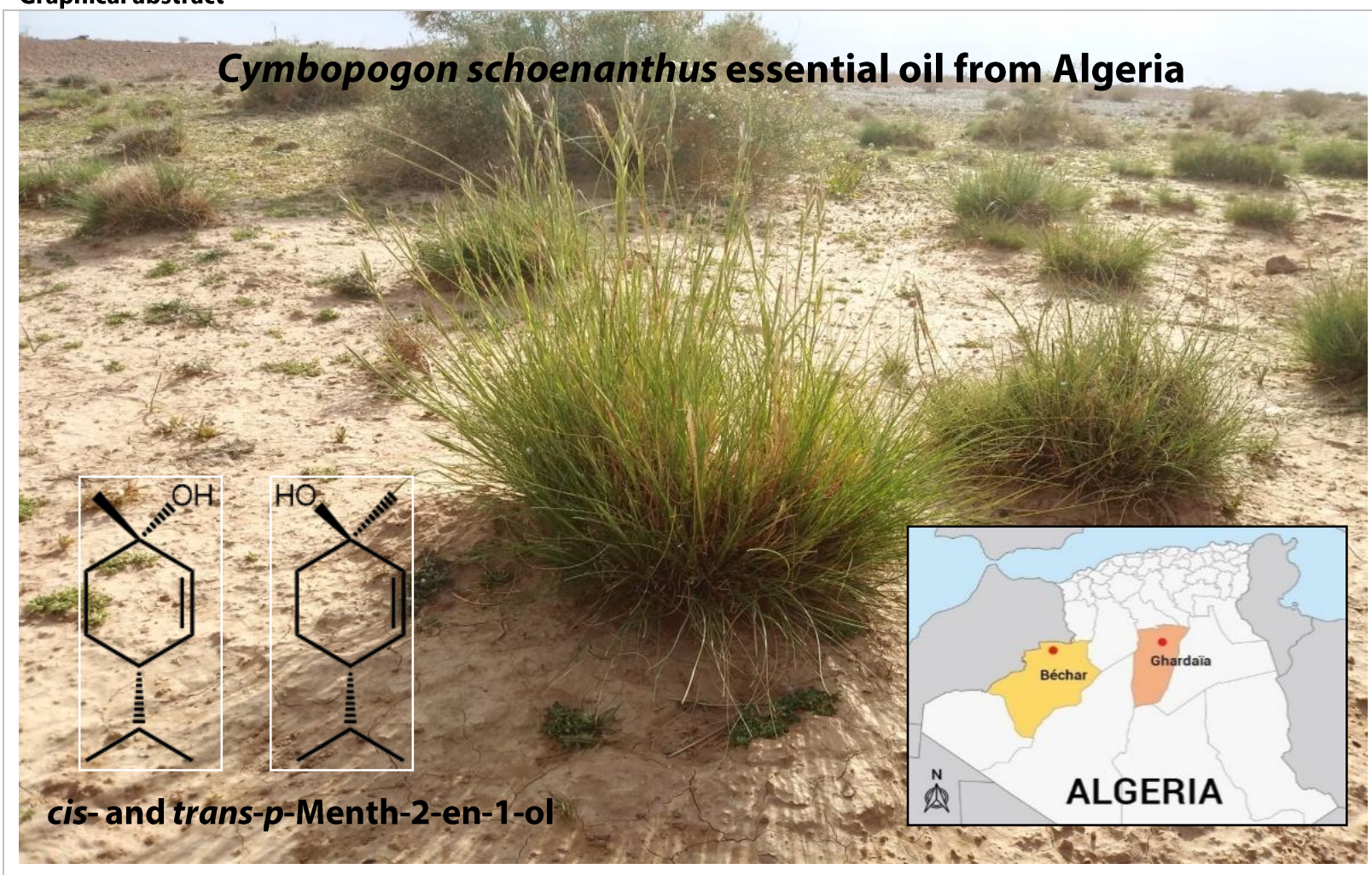

